\title{
RADIOLOGÍA
}

www.elsevier.es/rx

ORIGINAL

\section{Utilidad de la ecografía en la evaluación de los nódulos tiroideos}

\section{Carolina Franco Uliaque ${ }^{\mathrm{a}, *}$, Francisco Javier Pardo Berdún ${ }^{\mathrm{a}}$, Ricardo Laborda Herrero ${ }^{\mathrm{b}}$ y Carmen Pérez Lórenz ${ }^{a}$}

\author{
a Servicio de Radiodiagnóstico, Centro Médico de Especialidades Ramón y Cajal, Hospital Universitario Miguel Servet, \\ Zaragoza, España \\ ${ }^{\text {b } C e n t r o ~ U n i v e r s i t a r i o ~ d e ~ l a ~ D e f e n s a, ~ Z a r a g o z a, ~ E s p a n ̃ a ~}$
}

Recibido el 15 de septiembre de 2015; aceptado el 6 de junio de 2016

Disponible en Internet el 25 de julio de 2016

\section{PALABRAS CLAVE Nódulo tiroideo; Ecografía; Biopsia}

\begin{abstract}
Resumen
Objetivo: Evaluar la capacidad diagnóstica de la ecografía para diferenciar nódulos tiroideos benignos y malignos, y su utilidad para evitar procedimientos invasivos innecesarios.

Pacientes y métodos: De enero de 2012 a diciembre de 2014 se realizaron 321 procedimientos de punción-aspiración con aguja fina (PAAF) a 302 pacientes remitidos por el Servicio de Endocrinología del Centro Medico de Especialidades, seleccionados bajo los criterios de la Guía de la Asociación Americana de Endocrinología Clínica y de la Guía de la Asociación Americana del Tiroides. Se analizaron las siguientes características ecográficas: localización, tamaño, morfología, contorno, consistencia, ecoestructura, ecogenicidad, calcificaciones y vascularización. Se realizó un análisis univariante y multivariante para investigar la relación entre los hallazgos ecográficos y el cáncer de tiroides.

Resultados: La prevalencia de malignidad en nuestra población de estudio es del 5,92\%. Los hallazgos ecográficos que son estadísticamente significativos y están asociados a una mayor probabilidad de malignidad son la presencia de microcalcificaciones, la vascularización central y la hipoecogenicidad. Los hallazgos asociados a un menor riesgo son la presencia de halo, la existencia de áreas de degeneración coloide y la heterogeneidad de los nódulos.

Conclusión: Según nuestro estudio, la indicación de realizar PAAF no debería basarse en el tamaño del nódulo, sino en la presencia de hallazgos ecográficos sospechosos de malignidad de acuerdo con las estimaciones de nuestro modelo estadístico. De esta forma sugerimos evitar realizar PAAF innecesarias en nódulos que no presenten dichas características.

(c) 2016 SERAM. Publicado por Elsevier España, S.L.U. Todos los derechos reservados.
\end{abstract}

\footnotetext{
* Autor para correspondencia.

Correo electrónico: caroluliaque@hotmail.com (C. Franco Uliaque).
} 


\section{KEYWORDS}

Thyroid nodule; Ultrasonography; Biopsy

\section{Usefulness of ultrasonography is the evaluation of thyroid nodules}

\begin{abstract}
Objective: To evaluate the diagnostic capacity of ultrasonography (US) for differentiating between malignant and benign thyroid nodules and its usefulness in obviating unnecessary invasive procedures.

Patients and methods: From January 2012 through December 2014, a total of 321 fine-needle aspiration biopsy (FNAB) procedures were done in 302 patients selected according to the criteria recommended by the American Association of Clinical Endocrinology guidelines and the American Thyroid Association guidelines. We analyzed the following characteristics on US: location, size, morphology, contour, consistency, echostructure, echogenicity, calcifications, and vascularization. We used univariate and multivariate analyses to investigate the relationship between the US findings and thyroid cancer.

Results: The prevalence of malignancy in our study population was $5.92 \%$. The US findings that were significantly associated with a greater probability of malignancy were microcalcifications, central vascularization, and hypoechogenicity. The US findings that were associated with a lower risk of malignancy were areas of colloid degeneration and nodule heterogeneity.

Conclusion: Our results suggest that decisions about whether to perform FNAB should be based on the presence of suspicious US findings found with our statistic model rather than on the size of the nodule. Thus, unnecessary FNAB procedures on nodules without suspicious US characteristics can be avoided.
\end{abstract}

(c) 2016 SERAM. Published by Elsevier España, S.L.U. All rights reserved.

\section{Introducción}

La ecografía de cuello es una prueba diagnóstica de primera elección, ampliamente usada en las enfermedades tiroideas. Se estima que su utilización podría demostrar la existencia de nódulos tiroideos hasta en un $67 \%$ de la población, de los que serían malignos aproximadamente el $5 \%^{1}$. La ecografía tiene un papel imprescindible en la evaluación del tiroides, pues permite calcular su tamaño, diferenciar entre nódulos (único o múltiples) y una afectación difusa, sin o con nódulos. Sin embargo, aunque se ha intentado determinar qué características ecográficas diferencian los nódulos malignos de los benignos, actualmente no existe consenso sobre qué lesiones deben ser biopsiadas. Existen diversas guías de actuación en el manejo del nódulo tiroideo que proponen distintas estrategias, pero es necesario unificar criterios ${ }^{2}$. Ante este problema, hay que establecer qué hallazgos ecográficos indican que el nódulo es maligno o benigno con un valor predictivo aceptable, para poder decidir y priorizar qué nódulos se someterán a punción-aspiración con aguja fina (PAAF), pues a pesar de ser este el mejor método para diferenciar entre benignidad y malignidad, dada su alta sensibilidad y especificidad, es un procedimiento invasivo que no proporciona información inmediata y está sujeto a las incertidumbres o errores del muestreo y del análisis ${ }^{1,3,4}$.

Los objetivos de este estudio son analizar la capacidad diagnóstica del ultrasonido para diferenciar entre nódulos tiroideos benignos y malignos, determinando cuáles son los hallazgos ecográficos individuales o en asociación que pueden considerarse como factores de alto riesgo de malignidad, y valorar su utilidad para evitar procedimientos invasivos innecesarios.

\section{Material y métodos}

Declaramos expresamente nuestra adhesión a la declaración de Helsinki y se obtuvieron los consentimientos informados necesarios para realizar las exploraciones de todos los pacientes.

\section{Pacientes}

En este estudio retrospectivo y observacional se incluyeron 302 pacientes evaluados entre enero de 2012 y diciembre de 2014, con un total de 321 nódulos tiroideos diagnosticados, de los cuales 263 correspondían a mujeres, con una edad media de 57 años (rango: 21 a 87 años), y 58 correspondían a varones, cuya edad media era de 59 años (rango: 23 a 79 años). Se incluyeron en el estudio todos los pacientes remitidos por el Servicio de Endocrinología del Centro Medico de Especialidades, durante los 3 años referidos, para la realización de PAAF tiroidea, seleccionados bajo los criterios de la Guía de la Asociación Americana de Endocrinología Clínica y de la última versión de la Guía de la Asociación Americana del Tiroides ${ }^{5-7}$, según las cuales se recomienda biopsiar:

- Los nódulos mayores de $1 \mathrm{~cm}$ con patrón ecográfico de alta sospecha, cuyo riesgo de malignidad estimado es mayor del 70-90\%, incluyendo nódulos hipoecoicos sólidos o con componente parcial quístico, con una o más de las siguientes características ecográficas: márgenes irregulares (infiltrantes, espiculados o microlobulados), microcalcificaciones, forma más alta que ancha, calcificaciones en anillo interrumpidas por un pequeño componente de partes blandas hipoecoico y evidencia de extensión extratiroidea. 
- Los nódulos mayores de $1 \mathrm{~cm}$ con patrón ecográfico de sospecha intermedia, cuyo riesgo de malignidad estimado es del $10-20 \%$, incluyendo nódulos sólidos hipoecoicos con márgenes lisos sin microcalcificaciones, extensión extratorácica ni forma más alta que ancha.

- Los nódulos mayores de 1,5cm con patrón ecográfico de baja sospecha, cuyo riesgo de malignidad estimado es del 5-10\%, incluyendo nódulos isoecoicos o hiperecoicos sólidos o parcialmente quísticos con áreas sólidas excéntricas, sin microcalcificaciones, márgenes irregulares ni extensión extratiroidea, ni forma más alta que ancha.

- Los nódulos mayores de $2 \mathrm{~cm}$ con patrón ecográfico de muy baja sospecha, cuyo riesgo de malignidad estimado es menor del $3 \%$, incluyendo nódulos parcialmente quísticos o espongiformes, sin características ecográficas descritas en los patrones de baja, intermedia y alta sospecha.

- Todos aquellos nódulos, independientemente del tamaño, que hayan presentado crecimiento significativo en los controles ecográficos o sean de pacientes con antecedentes familiares o factores de alto riesgo.

Se excluyeron del estudio aquellos pacientes cuyo nódulo a puncionar era ecográficamente anecoico, y por tanto de naturaleza líquida.

En caso de existir múltiples nódulos, se realiza PAAF de todos los que presenten características ecográficas sospechosas de malignidad, porque si solamente puncionáramos el nódulo dominante dejaríamos de diagnosticar algún cáncer ${ }^{8}$. A todos los pacientes se les realizó ecografía (modo B y Doppler color), elastografía y PAAF ecodirigida. El anatomopatólogo se basó en la clasificación Bethesda-2007 ${ }^{9}$ para realizar el diagnóstico citológico. En los casos en que se obtuvo una citología de tipo I o no diagnóstica o insatisfactoria se repitió la $\mathrm{PAAF}$, y si se obtuvo el mismo resultado se realizó control ecográfico en 6 meses, con nueva valoración. Todos los pacientes cuya segunda PAAF fue nula fueron excluidos del estudio. Cuando en la PAAF se obtuvo una citología benigna o tipo ॥ (nódulo folicular benigno, tiroiditis linfocitaria y tiroiditis granulomatosa), con un riesgo de malignidad del $0-3 \%$, se realizó seguimiento clínico. En las categorías III o indeterminado (atipia de significado indeterminado y lesión folicular de significado indeterminado), con un riesgo de malignidad del $15-30 \%$, tipo ı o proliferación/neoplasia folicular, tipo $v$ o sospechoso de malignidad (carcinoma papilar, carcinoma medular, metástasis y linfoma), con un riesgo de malignidad del $60-75 \%$, y tipo vı o maligno (carcinoma papilar, carcinoma pobremente diferenciado, carcinoma medular, carcinoma anaplásico, carcinoma de células escamosas, carcinoma con características mixtas, carcinoma metastático, linfoma no Hodgkin), se realizó cirugía con tiroidectomía parcial o total, y posterior análisis anatomopatológico de la pieza quirúrgica.

\section{Ecografía tiroidea convencional}

Las ecografías tiroideas fueron realizadas por dos radiólogos, con 35 y 12 años de experiencia en ecografía, utilizando un ecógrafo Siemens Acuson Antares (Siemens Medical Solutions, Mountain View, CA, USA) equipado con un transductor lineal multifrecuencia de 8-12 MHz, con Doppler color y elastografía. Todas las PAAF las practicó un solo radiólogo con experiencia de 25 años en la técnica. Se empleó una aguja de calibre 21-22 G conectada a una jeringa de 20 cc y un mecanismo de aspiración (Cameco Medical Ltd London NW6 $2 \mathrm{BP})$, con visualización del nódulo mediante ecografía.

Se analizaron los siguientes parámetros para realizar la caracterización de cada nódulo estudiado:

1. Localización: los nódulos pueden encontrarse en el lóbulo tiroideo derecho, el lóbulo tiroideo izquierdo y el istmo.

2. Presencia o ausencia de otros nódulos tiroideos: puede tratarse de un nódulo único o de un nódulo en el contexto de un bocio multinodular.

3. Tamaño: los nódulos se clasifican en dos grupos en función de su tamaño, con eje mayor $<20 \mathrm{~mm}$ y $\geq 20 \mathrm{~mm}$.

4. Morfología: los nódulos pueden ser redondos, más anchos que altos y más altos que anchos.

5. Contorno: se distingue entre nódulos rodeados por un halo hipoecoico, nódulos bien delimitados y nódulos de márgenes mal definidos.

6. Consistencia: se incluyeron en el estudio nódulos sólidos y mixtos (aquellos con abundantes áreas de degeneración coloide), y se excluyeron las lesiones de naturaleza únicamente quística.

7. Ecoestructura: homogéneos cuando la estructura es uniforme y heterogéneos si presentan distintas ecogenicidades.

8. Ecogenicidad: se comparó la ecogenicidad de cada nódulo con la del parénquima tiroideo circundante. Se distinguieron cuatro tipos: hiperecoico, si es más ecogénico que el tejido tiroideo; isoecoico, cuando es similar; hipoecoico, si de forma generalizada es menor; y mixto, cuando existen áreas hipoecoicas, isoecoicas e hiperecoicas en el mismo nódulo, sin predominar ninguna.

9. Vascularización: con el Doppler color se definen tres patrones. Tipo ı: si presenta escaso flujo vascular; tipo II: si existe vascularización de predominio periférico (fig. 1); y tipo III: cuando predomina el flujo intranodular central (fig. 2).

10. Presencia o ausencia de calcificaciones intranodulares: en caso de existir calcificaciones, diferenciamos entre microcalcificaciones (fig. 3), descritas como imágenes hiperecogénicas de diámetro $\leq 2 \mathrm{~mm}$ con o sin sombra acústica posterior, y calcificaciones groseras (el resto).

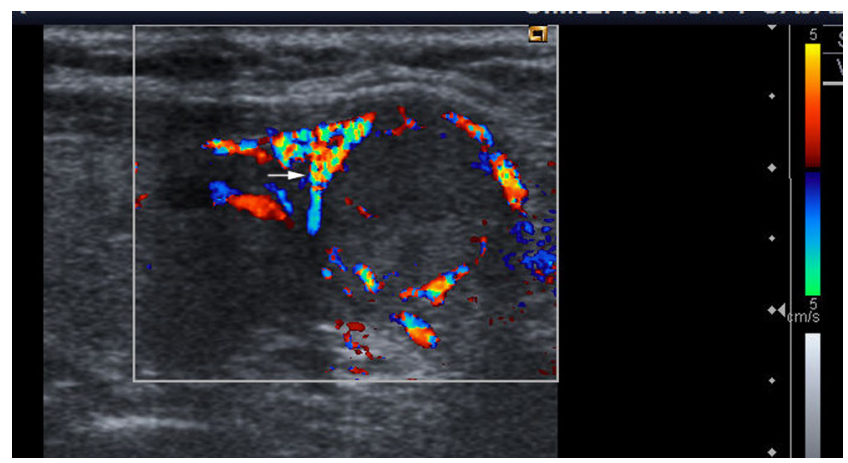

Figura 1 Ecografia tiroidea con Doppler color, corte transversal. Se muestra un nódulo tiroideo con patrón vascular tipo II, con vasos periféricos (flecha), en el contexto de hiperplasia nodular tiroidea. 


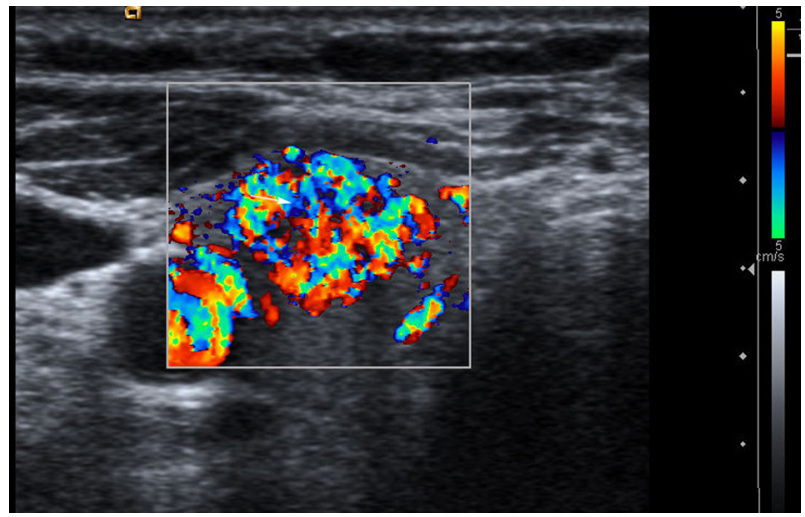

Figura 2 Ecografia tiroidea con Doppler color, corte transversal. Se muestra un nódulo tiroideo con patrón vascular tipo III, con vascularización central aumentada (flecha), en un nódulo tiroideo diagnosticado de carcinoma papilar.

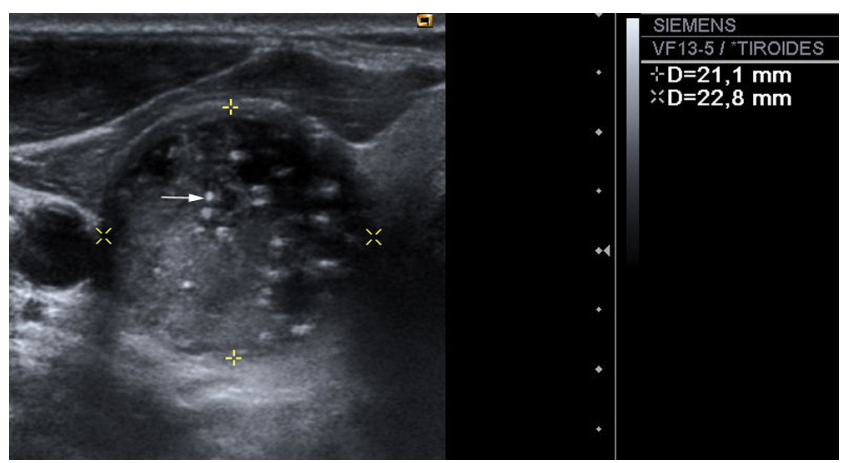

Figura 3 Ecografía tiroidea en modo $B$, corte transversal. Se muestra un nódulo tiroideo con múltiples microcalcificaciones dispersas (flecha) ocupando toda la lesión, diagnosticado de carcinoma papilar.

\section{Análisis estadístico}

Analizamos la probabilidad de que un nódulo tiroideo sea maligno en función de cada uno de los signos ecográficos en modo $B$ y Doppler color que se han descrito en el apartado anterior. Para ello utilizamos variables dicotómicas que reflejan la presencia o no del signo ecográfico analizado, y estimamos un modelo de regresión logística que proporciona un coeficiente $\beta$ que relaciona cada variable con la probabilidad de que el nódulo analizado sea maligno, y el valor de $\mathrm{p}$ asociado. Asimismo, se calculan las odds ratios (OR) y una medida del ajuste total del modelo (pseudo- $\mathrm{R}^{2}$ ).

Estudiamos la significatividad de cada variable explicativa por separado (análisis univariante) y de forma conjunta (análisis multivariante). Las variables explicativas consideradas en el modelo multivariante son aquellas que en el análisis univariante son significativas. De esta forma, consideramos las siguientes variables explicativas dicotómicas en el análisis multivariante: 1) si el nódulo es múltiple o no; 2) si hay presencia de halo o no; 3) si el nódulo es heterogéneo o no; 4) si presenta microcalcificaciones o no; 5) si hay áreas de degeneración coloide o $\mathrm{n}$; 6) si existe hipoecogenicidad o no; y 7) si la vascularización es central o no.

En nuestro análisis empírico hacemos especial hincapié en la capacidad de los modelos estimados para clasificar correctamente los nódulos en malignos o benignos. Con el fin de comprobar la efectividad del modelo en la clasificación de los nódulos, construimos una tabla de clasificación en la que se cruza el verdadero valor de cada nódulo en observación (1 o maligno, 0 o benigno) con la predicción que el modelo considerado asigna a dicho nódulo. Esta predicción se hace con respecto a un valor arbitrario de $\mathrm{P}_{0}$, de modo que un nódulo es catalogado como maligno o benigno si la probabilidad de malignidad que le asigna el modelo, $\mathrm{P}_{\text {estimada }}$, es superior o inferior a $P_{0}$, respectivamente. A partir de la tabla de clasificación calculamos la sensibilidad y la especificidad de cada modelo, así como la probabilidad de que la enfermedad esté presente cuando el resultado de la prueba diagnóstica es positivo para enfermedad, o valor positivo predictivo (VPP), y la probabilidad de que la enfermedad está ausente cuando el resultado de la prueba diagnóstica es negativo para enfermedad, o valor predictivo negativo (VPN). Repitiendo este proceso para todo valor posible de $\mathrm{P}_{0}$ obtenemos unos pares de sensibilidad y especificidad, que se representan en la curva ROC. Finalmente, se calcula el área bajo la curva ROC para cada modelo, interpretada como la probabilidad de clasificar correctamente a un paciente elegido al azar, y se representa para el caso multivariante.

Para los cálculos estadísticos se ha utilizado el programa Stata.

\section{Resultados}

El estudio incluyó 321 nódulos tiroideos, de los cuales 201 obtuvieron un resultado citológico de benignidad (Bethesda II) en la PAAF, y no se realizó ninguna prueba diagnóstica más. En 32 ocasiones recibimos un resultado no diagnóstico (Bethesda I) de las dos punciones realizadas, y en todos estos casos no se observaron cambios significativos en el control ecográfico realizado a los 6 meses, por lo que se pautó posteriormente un control evolutivo anual. Estos 32 nódulos fueron eliminados del análisis para no alterar los resultados. Los 88 nódulos restantes obtuvieron en la PAAF, mediante análisis citológico de la muestra, resultados Bethesda III, IV, $v$ y vI, y todos fueron intervenidos quirúrgicamente. De ellos, 3 nódulos se clasificaron como Bethesda III en la PAAF y su diagnóstico final tras la cirugía fue de hiperplasia nodular tiroidea; 67 nódulos se clasificaron como Bethesda IV tras la PAAF, de los cuales solamente 6 fueron cáncer en el diagnóstico definitivo; 9 nódulos obtuvieron un resultado de sospecha de malignidad en la PAAF, y de ellos la cirugía confirmó la malignidad en 4; y 9 nódulos se clasificaron como Bethesda vI o malignos en la PAAF y se confirmaron todos ellos tras la cirugía. En total, 19 nódulos obtuvieron un diagnostico final de malignidad, lo que representa un 5,92\% del total, con los siguientes diagnósticos histológicos: 12 nódulos con carcinoma papilar, 3 con variante folicular de carcinoma papilar, 2 con carcinoma folicular y 1 con carcinoma pobremente diferenciado. Los 69 nódulos tiroideos intervenidos restantes obtuvieron un diagnóstico histológico de benignidad: 54 nódulos correspondieron a hiperplasia nodular, 6 adenomas foliculares, 5 tiroiditis de Hashimoto, 4 tiroiditis crónica no Hashimoto y 2 adenomas de células de Hürtle.

En la tabla 1 se detalla la frecuencia absoluta de las características de los pacientes (sexo y edad) y de los parámetros ecográficos seleccionados para el total de la 
Tabla 1 Frecuencias absolutas de variables explicativas en función del diagnóstico de los nódulos

\begin{tabular}{|c|c|c|c|c|}
\hline Variable explicativa & Categoría & $\begin{array}{l}\text { Nódulo tiroideo } \\
\text { maligno }\end{array}$ & $\begin{array}{l}\text { Nódulo tiroideo } \\
\text { benigno }\end{array}$ & Total \\
\hline \multirow[t]{2}{*}{ Sexo } & Hombre & 4 & 54 & 58 \\
\hline & Mujer & 15 & 248 & 223 \\
\hline \multirow[t]{2}{*}{ Edad } & $<60$ años & 7 & 129 & 136 \\
\hline & $>60$ años & 12 & 173 & 185 \\
\hline \multirow[t]{3}{*}{ Localización } & LTD & 9 & 151 & 160 \\
\hline & LTI & 9 & 127 & 136 \\
\hline & Istmo & 1 & 24 & 25 \\
\hline \multirow{4}{*}{$\begin{array}{l}\text { Presencia o ausencia de otros } \\
\text { nódulos tiroideos } \\
\text { Tamaño }\end{array}$} & Nódulo único & 11 & 95 & 106 \\
\hline & Bocio multinodular & 8 & 207 & 215 \\
\hline & Grande & 12 & 209 & 221 \\
\hline & Pequeño & 7 & 93 & 100 \\
\hline \multirow[t]{2}{*}{ Morfología } & Más alto que ancho & 3 & 19 & 22 \\
\hline & Más ancho que alto & 16 & 283 & 199 \\
\hline \multirow[t]{3}{*}{ Contorno } & Halo & 1 & 79 & 80 \\
\hline & Nódulo bien definido & 12 & 158 & 170 \\
\hline & Nódulo mal definido & 6 & 65 & 71 \\
\hline \multirow[t]{2}{*}{ Ecoestructura } & Heterogénea & 12 & 260 & 272 \\
\hline & Homogénea & 7 & 42 & 49 \\
\hline Presencia o ausencia de & No calcificaciones & 10 & 235 & 245 \\
\hline \multirow[t]{2}{*}{ calcificaciones intranodulares } & Microcalcificaciones & 5 & 32 & 37 \\
\hline & Calcificaciones groseras & 4 & 35 & 39 \\
\hline \multirow[t]{2}{*}{$\begin{array}{l}\text { Presencia o ausencia de áreas } \\
\text { de degeneración coloide }\end{array}$} & $\begin{array}{l}\text { Presencia de áreas de } \\
\text { degeneración coloide }\end{array}$ & 3 & 152 & 155 \\
\hline & $\begin{array}{l}\text { Ausencia de áreas de } \\
\text { degeneración coloide: nódulo } \\
\text { completamente sólido }\end{array}$ & 16 & 150 & 166 \\
\hline \multirow[t]{3}{*}{ Vascularización } & Nulo o escaso flujo vascular & 3 & 85 & 88 \\
\hline & De predominio periférico & 4 & 137 & 141 \\
\hline & Central & 12 & 80 & 92 \\
\hline \multirow{4}{*}{ Ecogenicidad } & Isoecogénico & 5 & 116 & 121 \\
\hline & Hipoecogénico & 14 & 148 & 162 \\
\hline & Ecogénico & 0 & 23 & 23 \\
\hline & Mixto & 0 & 15 & 15 \\
\hline
\end{tabular}

LTD: lóbulo tiroideo derecho; LTI: lóbulo tiroideo izquierdo.

muestra, así como en función de la malignidad o la benignidad de los nódulos tiroideos analizados.

La tabla 1 muestra que la malignidad es más frecuente en los nódulos con microcalcificaciones, vascularización central y diámetro anteroposterior mayor que transversal (más alto que ancho), y menos frecuente en nódulos en el contexto de un bocio multinodular, en nódulos heterogéneos, en nódulos con áreas de degeneración coloide y en nódulos rodeados por un halo hipoecoico.

\section{Análisis estadístico univariante de la malignidad de los nódulos tiroideos}

La tabla 2 presenta los resultados obtenidos para el análisis de regresión logística univariante.

En nuestra muestra no son factores significativos de malignidad la edad ni el sexo del paciente. Asimismo, no encontramos significativos la localización, el tamaño ni la morfología del nódulo.
Los hallazgos ecográficos que son estadísticamente significativos $(p<0,05)$ y están asociados a una mayor probabilidad de malignidad son la presencia de microcalcificaciones, vascularización central e hipoecogenicidad. Los hallazgos ecográficos que son significativos y están asociados a una menor probabilidad de malignidad, y por tanto son indicativos de benignidad, son la presencia de halo y de áreas de degeneración coloide y la heterogeneidad de los nódulos.

La OR alcanza sus mayores valores para la vascularización central y la presencia de microcalcificaciones. De esta forma, un paciente cuyo nódulo tiroideo se caracterice por tener una vascularización central o microcalcificaciones incrementa su riesgo con relación a otro paciente que no presenta dichas características en un factor igual a 3,71 y 3,09 , respectivamente. En sentido contrario, si un nódulo está rodeado por un halo o presenta áreas de degeneración coloide el riesgo se reduce en un factor de 6,66 y 5,55, respectivamente.

A pesar de que nuestro análisis univariante indica que existen variables significativamente relacionadas con la 
Tabla 2 Resultados del análisis de regresión logística para la detección de nódulos de tiroides malignos

\begin{tabular}{|c|c|c|c|c|c|c|}
\hline Característica & $\beta$ & OR & $\mathrm{p}$ & $\begin{array}{l}\text { LR } \\
\chi^{2}(1)\end{array}$ & $\mathrm{p}$ & Pseudo- $R^{2}$ \\
\hline Localización LTD & $-0,26$ & 0,77 & 0,59 & 0,29 & 0,58 & 0,00 \\
\hline Bocio multinodular & $-1,07$ & 0,33 & 0,02 & 5,05 & 0,04 & 0,04 \\
\hline Tamaño grande & $-0,31$ & 0,73 & 0,54 & 0,38 & 0,54 & 0,00 \\
\hline Forma más alta que ancha & 1,09 & 2,97 & 0,11 & 2,12 & 0,15 & 0,01 \\
\hline Presencia de halo & $-1,88$ & 0,15 & 0,07 & 5,68 & 0,01 & 0,04 \\
\hline Ecoestructura heterogénea & $-1,21$ & 0,30 & 0,01 & 5,11 & 0,02 & 0,04 \\
\hline Hipoecogenicidad & 1,10 & 3,01 & 0,04 & 4,81 & 0,03 & 0,03 \\
\hline Presencia de microcalcificaciones & 1,13 & 3,09 & 0,04 & 3,50 & 0,06 & 0,02 \\
\hline Presencia de calcificaciones groseras & 0,70 & 2,01 & 0,24 & 1,43 & 0,24 & 0,00 \\
\hline Presencia de áreas de degeneración coloide & $-1,72$ & 0,18 & 0,00 & 9,73 & 0,00 & 0,07 \\
\hline Vascularización central & 1,31 & 3,71 & 0,00 & 7,36 & 0,00 & 0,05 \\
\hline Sexo hombre & 0,18 & 1,20 & 0,75 & 0,10 & 0,75 & 0,00 \\
\hline Edad $>60$ años & $-0,16$ & 0,85 & 0,74 & 0,74 & 0,61 & 0,00 \\
\hline
\end{tabular}

LR: likelihood ratio; LTD: lóbulo tiroideo derecho; OR: odds ratio.

malignidad del nódulo tiroideo, el estudio de la capacidad predictiva de los modelos estimados refleja que ninguna variable considerada de forma aislada permite predecir adecuadamente la malignidad de un nódulo tiroideo, porque la bondad del ajuste de los modelos medida por el pseudo- $\mathrm{R}^{2}$ es reducida y por la dificultad de obtener valores elevados de sensibilidad y especificidad de forma conjunta. En la tabla 2 puede verse que en ningún modelo el pseudo- $\mathrm{R}^{2}$ supera el $10 \%$. La curva ROC muestra una pobre capacidad predictiva de las variables explicativas: el área bajo la curva ROC es, para todos los modelos, muy cercana a 0,5 (clasificación de los nódulos tiroideos al «azar») y en ningún caso superior a 0,7 , lejos del mejor modelo posible (área bajo la curva ROC igual a 1). No existe ningún modelo cuya sensibilidad sea del $100 \%$ y tenga una especificidad superior al $0 \%$.

\section{Análisis multivariante de la malignidad de los nódulos tiroideos}

La tabla 3 presenta los resultados de la estimación del modelo de regresión logística multivariante. El modelo multivariante estimado preserva los signos de los coeficientes estimados en el análisis univariante. Es decir, la probabilidad de malignidad del nódulo tiroideo está positivamente relacionada con la presencia de microcalcificaciones, vascularización central e hipoecogenicidad, y negativamente relacionada con la presencia de halo, la presencia de áreas de degeneración coloide y la heterogeneidad de los nódulos.

Las variables que proporcionan una mayor OR son la vascularización central y la presencia de microcalcificaciones. En sentido contrario, las variables que tienen una menor OR son la hipoecogenicidad, la presencia de halo y la presencia de áreas de degeneración coloide.

De acuerdo con la estimación del modelo de regresión logística, un paciente de «máximo riesgo» cuyo nódulo tiroideo presenta microcalcificaciones, vascularización central, hipoecogenicidad, ausencia de halo y ausencia de áreas de degeneración coloide tiene una OR de 2,50. En el lado opuesto, un paciente de «mínimo riesgo» que presenta ausencia de microcalcificaciones, ausencia de vascularización central e hipoecogenicidad, combinados con la presencia de halo y de áreas de degeneración coloide, tiene una OR de 0,001.

Como era de esperar, el análisis de la capacidad de predicción del modelo multivariante mejora sustancialmente con relación al análisis univariante en términos de ajuste del modelo y de la capacidad predictiva de este. El pseudo- $\mathrm{R}^{2}$ aumenta de manera significativa hasta superar ligeramente el $23 \%$, de modo que se acepta el contraste de significatividad del modelo $(p=0)$. Tomando como referencia diferentes puntos de corte de la probabilidad de malignidad estimada, el modelo multivariante mejora sustancialmente los resultados del análisis univariante, sobre todo en cuanto a la

Tabla 3 Resultados del análisis de regresión logística para la detección de nódulos de tiroides malignos utilizando diversas variables explicativas

\begin{tabular}{lllll}
\hline Características & $\beta$ & OR & P & Pseudo-R \\
\hline Bocio multinodular & $-0,84$ & 0,44 & 0,11 & 0,23 \\
Presencia de halo & $-2,07$ & 0,12 & 0,06 \\
Ecoestructura heterogénea & $-1,04$ & 0,35 & 0,10 \\
Presencia de microcalcificaciones & 0,81 & 2,24 & 0,22 \\
Presencia de áreas de degeneración coloide & $-1,40$ & 0,24 & 0,04 \\
Hipoecogenicidad & 0,71 & 2,24 & 0,17 \\
Vascularización central & 0,81 & 2,02 & 0,19 \\
\hline
\end{tabular}

OR: odds ratio. 


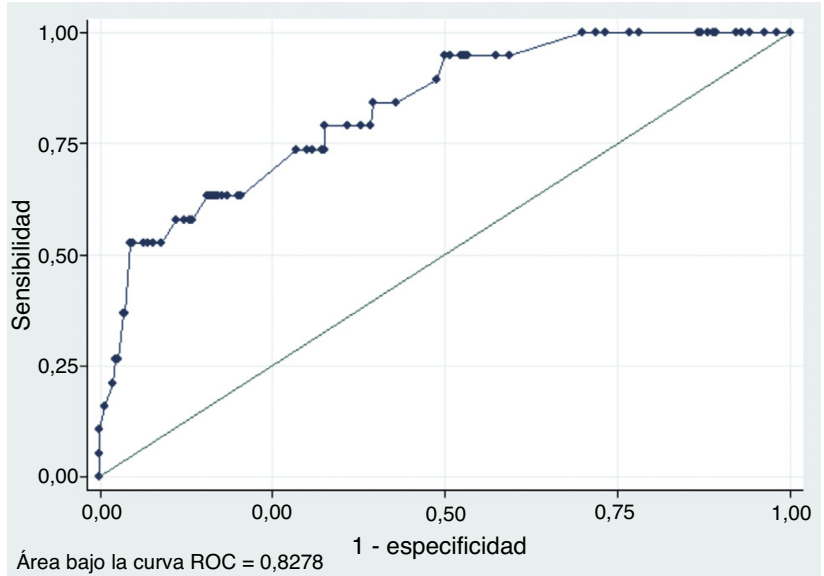

Figura 4 Curva ROC utilizando diversas variables explicativas de la probabilidad de malignidad de un nódulo tiroideo. La figura presenta la curva ROC asociada al modelo logístico multivariante que explica la probabilidad de malignidad de un nódulo tiroideo en función de los siguientes signos ecográficos en modo B y Doppler color: 1) si el nódulo es múltiple o no; 2) si hay presencia de halo o no; 3) si el nódulo es heterogéneo o no; 4) si existen microcalcificaciones o no; 5) si hay áreas de degeneración coloide o no; 6) si existe hipoecogenicidad o no; y 7) si la vascularización es central o no.

posibilidad de obtener, para una determinada sensibilidad, una especificidad más alta. La figura 4 muestra la curva ROC obtenida a partir del modelo, cuya área bajo la curva es 0,82 , muy superior a los valores alcanzados en el análisis univariante, lo que proporciona un argumento adicional de la bondad del modelo.

De acuerdo con el objetivo perseguido por este trabajo, mostramos los resultados de una posible estrategia a implementar a partir de la estimación del modelo bajo el supuesto de que es deseable una sensibilidad máxima para que ninguna neoplasia deje de diagnosticarse. La tabla 4 muestra que esta estrategia conservadora diagnostica correctamente los nódulos malignos, y a la vez permitió diagnosticar correctamente al menos 79 nódulos benignos. La sensibilidad del modelo es del $100 \%$ y la especificidad es del $28,15 \%$. La tabla 5 detalla la frecuencia relativa de las variables utilizadas en el modelo de regresión logística observadas en los 79 nódulos benignos que han sido calificados como tales según el modelo de regresión logística.
Tabla 5 Frecuencias relativas de variables explicativas de los nódulos clasificados como benignos a partir de la estrategia implementada ${ }^{a}$

\begin{tabular}{lc}
\hline Características & Porcentaje \\
\hline Bocio multinodular & $76 \%$ \\
Presencia de halo & $69 \%$ \\
Ecoestructura heterogénea & $91 \%$ \\
Presencia de microcalcificaciones & $0 \%$ \\
Presencia de áreas de degeneración coloide & $68 \%$ \\
Hipoecogenicidad & $18 \%$ \\
Vascularización central & $10 \%$ \\
\hline
\end{tabular}

a La estrategia implementada a partir de la estimación del modelo asume el supuesto de que es deseable una sensibilidad máxima para que ninguna neoplasia deje de diagnosticarse.

\section{Discusión}

Los resultados obtenidos en nuestro estudio y el posterior análisis estadístico concuerdan con parte de la evidencia empírica de estudios anteriores, aunque también presentan discrepancias con parte de la literatura previa. En nuestra muestra, casi un $6 \%$ de los pacientes tenían cáncer de tiroides, lo que constituye un porcentaje similar al descrito por Papini et al. ${ }^{10}$ y lannuccilli et al. ${ }^{11}$, pero considerablemente menor que el reportado en la mayoría de los artículos ${ }^{1,12-15}$, que lo elevan hasta el entorno del $14 \%$.

En nuestro estudio, los hallazgos ecográficos que son estadísticamente significativos y van asociados a una mayor probabilidad de malignidad son la hipoecogenicidad, la presencia de microcalcificaciones y el patrón de vascularización central. Por otro lado, los hallazgos ecográficos que son estadísticamente significativos y van asociados a una mayor probabilidad de benignidad son la heterogeneidad, la presencia de áreas coloides y la existencia de un halo hipoecoico. Nuestros resultados están en línea con alguna de la evidencia empírica previa.

lannuccilli et al. ${ }^{11}$ afirman que el único criterio estadísticamente significativo es la presencia de microcalcificaciones, y Papini et al. ${ }^{10}$ encuentran que los hallazgos ecográficos que tienen poder predictivo de malignidad, conforme a un análisis de regresión logística, son el patrón vascular intranodular, las microcalcificaciones y los márgenes irregulares o lobulados del nódulo, pero no encuentran que la presencia de halo hipoecoico sea indicativa de benignidad.

Tabla 4 Clasificación de los nódulos a partir de la estrategia implementada ${ }^{a}$

\begin{tabular}{|c|c|c|c|}
\hline & & \multicolumn{2}{|c|}{ Realidad } \\
\hline & & Nódulo maligno & Nódulo benigno \\
\hline \multirow[t]{2}{*}{ Predicción modelo regresión logística } & Nódulo maligno & 19 & 194 \\
\hline & Nódulo benigno & 0 & 76 \\
\hline Sensibilidad & $100 \%$ & & \\
\hline Especificidad & $28,15 \%$ & & \\
\hline
\end{tabular}


Kwak et al. ${ }^{16}$ hallan una asociación significativa con malignidad de la presencia de componente sólido, microcalcificaciones, márgenes irregulares o microlobulados, hipoecogenicidad y diámetro anteroposterior mayor que el trasversal.

Frates et al. ${ }^{13}$ refieren que el hallazgo ecográfico con mayor sensibilidad es la composición sólida del nódulo, pero con un VPP muy bajo, y que el hallazgo con mayor VPP son las microcalcificaciones.

Para Moon et al. ${ }^{15}$, los hallazgos ecográficos sospechosos de malignidad estadísticamente significativos son la forma más alta que ancha, los márgenes espiculados, una marcada hipoecogenicidad, la presencia de microcalcificaciones y macrocalcificaciones; y de benignidad, la apariencia espongiforme del nódulo y la isoecogenicidad. Estos autores conceden mucha importancia a la forma del nódulo y afirman que ser más alto que ancho es muy específico de malignidad, porque estos nódulos malignos crecen a través de los planos de tejidos normales, mientras que los nódulos benignos lo hacen paralelos a los planos de tejido normal.

Para Rago y Vitti ${ }^{17}$, los hallazgos ecográficos asociados a malignidad son las microcalcificaciones, la hipoecogenicidad, los márgenes irregulares, la ausencia de halo, el aspecto sólido, la vascularización intranodular y la forma más alta que ancha, variantes que individualmente tienen escaso valor predictivo, pero que cuando aparecen simultáneamente en un mismo nódulo aumentan la especificidad de la ecografía, aunque la sensibilidad sigue siendo inaceptablemente baja.

Kim et al. ${ }^{18}$, tras analizar 155 nódulos tiroideos no palpables, coinciden con nosotros en no encontrar diferencias estadísticamente significativas respecto al tamaño entre nódulos benignos y malignos. Proponen como indicaciones para realizar una PAAF la aparición de al menos uno de los cuatro criterios ecográficos que, según su estudio, son sugestivos de malignidad: microcalcificaciones, márgenes irregulares o microlobulados, marcada hipoecogenicidad interna del nódulo y morfología más alta que ancha. A diferencia de nosotros, no incluyen el aumento de la vascularización central como hallazgo sospechoso.

Es importante señalar, como limitaciones de nuestro estudio, que los distintos hallazgos ecográficos considerados de manera individual no permiten obtener conjuntamente una altas sensibilidad y especificidad en el diagnóstico de cáncer de tiroides. Bajo el supuesto de que es deseable una sensibilidad máxima para que ninguna neoplasia deje de diagnosticarse, los modelos univariantes alcanzarían una sensibilidad del $100 \%$ a costa de una especificidad del $0 \%$. De esta forma, los modelos univariantes no reducirían el número de PAAF realizadas. Por el contrario, la estimación del modelo multivariante nos ha permitido alcanzar una sensibilidad del $100 \%$ en el análisis dentro de la muestra con una especificidad del 28,15\%. Así pues, nuestro modelo ahorraría realizar la PAAF a 76 nódulos de los 270 benignos, lo que constituye una mejora con relación a la situación actual, en la que se practicó PAAF a todos los nódulos benignos. Asimismo, es preciso comprobar la robustez de nuestros resultados en un análisis fuera de la muestra, para lo que se requiere ampliar nuestra base de pacientes.

En consecuencia, observamos que nuestro estudio coincide con la mayoría de los estudios de la literatura en la determinación de cuáles son los hallazgos ecográficos sospechosos de malignidad, siendo los más significativos la presencia de microcalcificaciones, la vascularización central y la hipoecogenicidad, así como los hallazgos ecográficos asociados a benignidad, siendo los más significativos la presencia de áreas coloides, la presencia de halo, la existencia de bocio multinodular y la heterogeneidad de los nódulos.

A partir de los resultados del modelo estimado de regresión logística destacamos que es necesaria la presencia conjunta de varios hallazgos ecográficos para realizar un mejor diagnóstico de la malignidad de un nódulo tiroideo, permitiendo además eliminar la realización de PAAF en el mayor número de casos posible. Este resultado es congruente con los criterios vigentes de la Guía de la Asociación Americana de Endocrinología Clínica y de la última versión de la Guía de la Asociación Americana del Tiroides para biopsiar nódulos tiroideos.

En conclusión, en los nódulos que no tienen microcalcificaciones, vascularización central ni hipoecogenicidad, y que presentan halo y áreas de degeneración coloide, no está indicada la realización de PAAF porque la probabilidad de malignidad es muy baja.

\section{Responsabilidades éticas}

Protección de personas y animales. Los autores declaran que para esta investigación no se han realizado experimentos en seres humanos ni en animales.

Confidencialidad de los datos. Los autores declaran que en este artículo no aparecen datos de pacientes.

Derecho a la privacidad y consentimiento informado. Los autores declaran que en este artículo no aparecen datos de pacientes.

\section{Autoría}

1. Responsable de la integridad del estudio: CFU.

2. Concepción del estudio: CFU, JPB.

3. Diseño del estudio: CFU.

4. Obtención de los datos: CFU, JPB.

5. Análisis e interpretación de los datos: CFU, JPB, RLH.

6. Tratamiento estadístico: CFU, RLH.

7. Búsqueda bibliográfica: CFU, JPB, CPL.

8. Redacción del trabajo: CFU, JPB, RLH, CPL.

9. Revisión crítica del manuscrito con aportaciones intelectuales relevantes: JPB, CPL.

10. Aprobación de la versión final: CFU, JPB, RLH, CPL.

\section{Conflicto de intereses}

Los autores declaran no tener ningún conflicto de intereses.

\section{Bibliografía}

1. Rago T, Santini F, Scutari M, Pinchera A, Vitti P. Elastography: new developments in ultrasound for predicting malignancy in thyroid nodules. J Clin Endocrinol Metab. 2007;92:2917-22.

2. Manso S, Velasco MJ. Valor actual de la ecografía en la caracterización de los nódulos tiroideos. Revisión de las últimas guías clínicas de actuación. Radiología. 2015;57:248-58. 
3. Lyshchik A, Higashi T, Asato R, Tanaka S, Ito J, Mai JJ, et al. Thyroid gland tumor diagnosis at US elastography. Radiology. 2005;237:202-11.

4. Kagoya R, Monobe H, Tojima H. Utility of elastography for differential diagnosis of benign and malignant thyroid nodules. Otolaryngol Head Neck Surg. 2010;143:230-4.

5. Gharib H, Papini E, Valcavi R, Baskin HJ, Crescenzi A, Dottorini ME, et al. American Association of Clinical Endocrinologists and Associazione Medici Endocrinologi medical guidelines for clinical practice for the diagnosis and management of thyroid nodules. Endocrinol Pract. 2006;12:63-102.

6. Cooper DS, Doherty GM, Haugen BR, Kloos RT, Lee SL, Mandel SJ, et al. Revised American Thyroid Association management guidelines for patients with thyroid nodules and differentiated thyroid cancer. Thyroid. 2009;19:1167-214.

7. Haugen BR, Alexander EK, Bible KC, Doherty GM, Mandel SJ, Nikiforov YE, et al., The American Thyroid Association Guidelines Task Force on Thyroid Nodules and Differentiated Thyroid Cancer. 2015 American Thyroid Association Management Guidelines for adult patients with thyroid nodules and differentiated thyroid cancer. Thyroid. 2016;26:1-133.

8. Kim MJ, Kim EK, Park SI, Kim BM, Kwak JY, Kim SJ, et al. USguided fine needle aspiration of thyroid nodules: indications, techniques, results. Radiographics. 2008;28:1869-89.

9. Cibas ES, Ali SZ. The Bethesda System for reporting thyroid cytopathology. Am J Clin Pathol. 2009;132:658-65.

10. Papini E, Guglielmi R, Bianchini A, Crescenzi A, Taccogna S, Nardi F, et al. Risk of malignancy in nonpalpable thyroid nodules: predictive value of ultrasound and color-Doppler features. J Clin Endocrinol Metab. 2002;87:1941-6.
11. lannuccilli JD, Cronan JJ, Monchick JM. Risk for malignancy of thyroid nodules as assessed by sonographic criteria: the need for biopsy. J Ultrasound Med. 2004;23:1455-64.

12. Moon HJ, Sung JM, Kim EK, Yoon JH, Youk JH, Kwak JY. Diagnostic performance of gray-scale US and elastography in solid thyroid nodules. Radiology. 2012;262:1002-13.

13. Frates MC, Benson CB, Charboneau JW, Cibas ES, Clark OH, Coleman BG, et al. Management of thyroid nodules detected at US: Society of Radiologists in Ultrasound consensus conference statement. Radiology. 2005;237:794-800.

14. Frates MC, Benson CB, Doubilet PM, Kunreuther E, Contreras M, Cibas ES, et al. Prevalence and distribution of carcinoma in patients with solitary and multiple thyroid nodules on sonography. J Clin Endocrinol Metab. 2006;91:3411-7.

15. Moon HJ, Jung SL, Lee JH, Na DG, Baek JH, Lee YH, et al. Benign and malignant thyroid nodules: US differentiation multicenter retrospective study. Radiology. 2008;247:762-70.

16. Kwak JY, Han KH, Yoon JH, Moon HJ, Son EJ, Park SH, et al. Thyroid imaging reporting and data system for US features of nodules: a step in establishing better stratification of cancer risk. Radiology. 2011;260:892-9.

17. Rago T, Vitti P. Role of thyroid ultrasound in the diagnostic evaluation of thyroid nodules. Best Pract Res Clin Endocrinol Metab. 2008;22:913-28.

18. Kim EK, Park CS, Chung WY, Oh KK, Kim DI, Lee JT, et al. New sonographic criteria for recommending fine needle aspiration biopsy of nonpalpable solid nodules of the thyroid. AJR Am J Roentgenol. 2002;178:687-91. 